\title{
Comprehensive lymphadenectomy and survival prediction in uterine serous cancer patients after surgery: a population-based analysis
}

Hui Li ( $\square$ lihui239@mail.sysu.com )

Sun Yat-sen Memorial Hospital https://orcid.org/0000-0001-6214-8308

\section{Xiaofei Xie}

Department of Gynecological Oncology, Sun Yat-sen Memorial Hospital, Sun Yat-sen University, Guangzhou 510120, People's Republic of China

\section{Yunyun Liu}

Department of Gynecological Oncology, Sun Yat-sen Memorial Hospital, Sun Yat-sen University, Guangzhou 510120, People's Republic of China

\section{Xiaoxin Huang}

Department of Gynecological Oncology, Sun Yat-sen Memorial Hospital, Sun Yat-sen University, Guangzhou 510120, People's Republic of China

\section{Haoliang Lin}

Department of Gynecological Oncology, Sun Yat-sen Memorial Hospital, Sun Yat-sen University, Guangzhou 510120, People's Republic of China

\section{Jing Li}

Department of Gynecological Oncology, Sun Yat-sen Memorial Hospital, Sun Yat-sen University, Guangzhou 510120, People's Republic of China

\section{Zhongqiu Lin}

Department of Gynecological Oncology, Sun Yat-sen Memorial Hospital, Sun Yat-sen University, Guangzhou 510120, People's Republic of China

\section{Research}

Keywords: uterine serous cancer, nomogram, overall survival, cancer-specific survival, comprehensive lymphadenectomy, SEER

Posted Date: February 26th, 2020

DOI: https://doi.org/10.21203/rs.2.24587/v1

License: (c) (i) This work is licensed under a Creative Commons Attribution 4.0 International License. Read Full License 
Version of Record: A version of this preprint was published at European Journal of Surgical Oncology on July 1st, 2020. See the published version at https://doi.org/10.1016/j.ejso.2020.04.055. 


\section{Abstract}

Objective Evidence on uterine serous cancer (USC) prognosis has been limited and inconclusive. We aim to explore the survival benefits of comprehensive lymphadenectomy in USC patients after surgery and develop a prognostic nomogram to predict survival.

Materials and Methods USC patients who had undergone hysterectomy between 2010 and 2015 were identified from Surveillance, Epidemiology and End Results (SEER) database. The relationship between the extent of lymphadenectomy and survival, including overall survival (OS) and cancer-specific survival (CSS), was estimated with Kaplan-Meier (K-M) analysis. Univariate and multivariate Cox regression analyses were utilized to determine the independent prognostic factors. A nomogram was then developed, calibrated and internally validated.

Results A total of 2853 patients were identified. K-M survival analysis revealed that patients with $\geq 12$ pelvic lymph nodes (PLNs) removed had significantly better OS and CSS than those without (both $P$ $<0.001)$. However, patients with $\geq 6$ para-aortic lymph nodes removed was not associated with similar survival benefits than patients without $(P>0.1)$. Multivariate analyses for $O S$ and CSS revealed that age, T stage, $\mathrm{N}$ stage, tumor size, adjuvant therapy and $\geq 12 \mathrm{PLNs}$ removed were independent prognostic factors (all $\mathrm{P}<0.05$ ) and were subsequently incorporated into the nomogram. The Harrell's C-index of the nomogram was significantly higher than that of the International Federation of Gynecology and Obstetrics staging system (OS: 0.739 vs 0.671 , $P<0.001$; CSS: 0.752 vs $0.695, P<0.001$ ). Furthermore, the nomogram was well calibrated with satisfactory consistency.

Conclusions Comprehensive pelvic lymphadenectomy should be recommended to USC patients for its survival benefits. And a nomogram has been developed to predict the survivals of USC patients after surgery.

\section{Introduction}

Endometrial cancer is the fourth commonest malignant disorder in women worldwide and the commonest gynecological cancer in developing countries. Most endometrial cancers (80\%) are earlystage, low-grade, endometrioid-type tumors, which generally have favorable prognosis, with a 5-year overall survival (OS) of more than $85 \%$ (1). In contrary, uterine serous cancer (USC), the commonest subtype of nonendometrioid cancer, is aggressive and resembles serous ovarian carcinoma in terms of its histology and pattern of spread $(2,3)$. Approximately $69-87 \%$ of USC cases have already spread outside the uterus at the time of diagnosis, even those apparently confined to the uterus $(1,4)$. Moreover, $31-80 \%$ of USC recurs within the first 2 years after initial treatment. Therefore, patients with USC have a poor 5 -year OS of only $18-27 \%$. In addition, it accounts for $40 \%$ of overall mortality, even though its incidence is only $10 \%(5-8)$.

Given the rarity of USC, only a few prospective trials and some small-scale retrospective studies have been carried out to inform treatment strategies. For early stages, including stage I and II, the potential 
curative treatment is complete surgical staging followed by adjuvant radiation and paclitaxel-platinum chemotherapy $(2,8-13)$. On the other hand, cytoreduction surgery combined with adjuvant radiotherapy and chemotherapy are recommended for advanced stages (stage III-IV) $(3,14,15)$. According to the International Federation of Gynecology and Obstetrics (FIGO) guidelines, USC patients are recommended to receive surgical staging, including total hysterectomy with bilateral salpingo-oophorectomy, comprehensive pelvic lymphadenectomy (PLND) and selective para-aortic lymphadenectomy (PALND) (16). However, the National Comprehensive Cancer Network (NCCN) guide indicates that USC patients should undergo complete PALND at the renal vein level(17). Although the diagnostic value of lymph node dissection has been established, its therapeutic effects are still highly controversial. Two large prospective randomized trials of PLND failed to show any survival benefits, but many retrospective investigators have drawn conclusions that PLND and the number of removed pelvic lymph nodes are independent prognostic factors for survival (18-26). A retrospective cohort study enrolled 55 patients with nonendometrioid cancer recommended PALND in addition to PLND (27), but another study with a larger sample size demonstrated that PALND failed to deliver survival benefits for patients with high-risk endometrial cancer (18). Currently, the FIGO staging system remains the most widely used prognostic tool, in which nonmetastatic USC patients are stratified based on the depth of myometrial invasion, extent of spread and lymph node involvement. However, the survival of patients within the same FIGO stage has been heterogenous $(8,16)$. We believe that other independent prognostic factors, such as age, tumor size and treatment-related factors are also predictive of patient survival after surgery $(2,4,8,16)$.

Nomograms have been widely accepted as reliable and intuitive prognostic tools that incorporate and quantify relevant prognostic factors (28). Nomograms have been demonstrated to generate more precise survival predictions than the traditional staging system (29). However, to the best of our knowledge, a nomogram for USC patients after surgery has never been developed. This study aims to explore the prognostic effects of comprehensive lymphadenectomy in USC patients after surgery and develop an effective nomogram to predict 3-and 5-year survival for USC patients after surgery based on the Surveillance, Epidemiology, and End Results (SEER) database.

\section{Materials And Methods}

\section{Data sources and study population}

National Cancer Institute's SEER database from 1975 to 2016 (Nov. 2018 submission) was queried, and all related data were extracted from the publicly available SEER ${ }^{\star}$ Stat software (version 8.3.6) and were exempt from institutional review board approval.

USC patients who had undergone at least total hysterectomy between 2010 and 2015 were included. On the other hand, patients with metastatic disease (M1), unknown pathologic stage or unknown lymph node dissection were excluded (Supplementary Fig. 1). 
Relevant clinicopathological variables, including age, race, grade, adjusted AJCC 7th stage, tumor size, chemotherapy, radiotherapy, lymphadenectomy, number of removed lymph nodes from PLND/PALND, and survival information were extracted from the SEER database.

\section{Statistical analysis}

Chi-square/Fisher's exact tests were used to compare the clinical characteristics between groups. KaplanMeier (KM) survival analysis was used to calculate the cumulative OS and CSS, and univariate and multivariate Cox regression analyses were applied to identify independent prognostic factors. Hazard ratios (HRs) and $95 \%$ confidence intervals $(95 \% \mathrm{Cls}$ ) of the risk factors were also calculated.

To develop a predictive nomogram, survival curves for different variables were generated by K-M estimates and compared using the log-rank test. Variables with $\mathrm{P}<0.05$ were entered into the multivariate Cox regression model. A nomogram was formulated with significant variables from multivariate analysis, and the final model was optimized based on the Akaike information criteria (AIC).

Internal validation of the nomogram was performed with 2500 bootstrap resampling. Harrell's concordance-index (C-index) was used to assess the discriminative performance. The value of the Cindex ranges from 0.5 to 1.0 , which indicates that the discriminative power of the nomogram ranges from random chance to perfect matching. The comparison of C-indices between two models was also reported. Calibration curves of the nomogram for 3-and 5-year survival were constructed to compare consistency between the predicted survival and observed survival after bias correction.

We classified the population into low-, intermediate- and high-risk subgroups according to the total risk scores in the nomogram. Respective KM survival curves in each FIGO stage were depicted to further illustrate the independent discrimination ability of the nomogram. All computations were carried out in SPSS 25.0 software (IBM Corp, NY, USA) and R 3.6.1. Two-sided P values $<0.05$ were considered statistically significant.

\section{Results}

\section{Patient characteristics}

The characteristics of the patients are presented in Table 1. A total of 2853 USC patients were included in this study and the mean follow-up time was 35.9 months. A total of 2427 patients underwent PLND, and the median count of removed pelvic lymph nodes was 12 (interquartile range [IQR]: 7-18). Among these patients, 577 (23.8\%) patients had pathologically positive pelvic lymph nodes, and no significant difference in the positive rate was found between the $\geq 12$ subgroup and the $<12$ subgroup $(24.1 \% \mathrm{vs}$ $23.4 \%, P=0.68)$. A total of 1643 patients underwent PLND + PALND, and the median count of removed para-aortic lymph nodes was 5 (IQR: 3-9). Interestingly, a significantly higher probability of para-aortic lymph node metastases was found in the $\geq 6$ subgroup than in the $<6$ subgroup $(21.1 \%$ vs $16.3 \%, P=$ 0.01, Supplementary Table 1). 
Table 1

Basic characteristic in USC patients stratified by lymph node dissection.

\begin{tabular}{|c|c|c|c|c|c|}
\hline \multirow[t]{2}{*}{ Characteristics } & Total & No LND & PLND only & $\begin{array}{l}\text { PLND + } \\
\text { PALND }\end{array}$ & \multirow[t]{2}{*}{$\begin{array}{l}P \\
\text { value }\end{array}$} \\
\hline & $\begin{array}{l}n=2853 \\
(\%)\end{array}$ & $\begin{array}{l}n=426 \\
(\%)\end{array}$ & $\begin{array}{l}n=784 \\
(\%)\end{array}$ & $n=1643(\%)$ & \\
\hline Age & & & & & 0.11 \\
\hline$<60$ & $436(15.3)$ & $57(13.4)$ & 108 (13.8) & $271(16.5)$ & \\
\hline$\geq 60$ & 2417 (84.7) & $369(86.6)$ & $676(86.2)$ & $1372(83.5)$ & \\
\hline Race & & & & & $0.09 *$ \\
\hline White & 1939 (68.0) & $285(66.9)$ & $525(67.0)$ & $1129(68.7)$ & \\
\hline Black & $651(22.8)$ & $109(25.6)$ & $183(23.3)$ & 359 (21.9) & \\
\hline Others & $251(8.8)$ & $29(6.8)$ & 70 (8.9) & $152(9.3)$ & \\
\hline Unknown & $12(0.4)$ & $3(0.7)$ & $6(0.8)$ & $3(0.2)$ & \\
\hline Stage & & & & & 0.37 \\
\hline Early (I\&II) & 1895 (66.4) & 287 (67.4) & $534(68.1)$ & $1074(65.4)$ & \\
\hline Late (IIIA-IVA) & $958(33.6)$ & 139 (32.6) & 250 (31.9) & $569(34.6)$ & \\
\hline T stage & & & & & $<0.001$ \\
\hline T1 & 1931 (67.7) & $241(56.6)$ & $536(68.4)$ & 1154 (70.4) & \\
\hline T2 & $330(11.6)$ & $51(12.0)$ & 89 (11.4) & $190(11.6)$ & \\
\hline T3 & $537(18.8)$ & 105 (24.6) & 149 (19.0) & $283(17.2)$ & \\
\hline T4 & $55(1.9)$ & $29(6.8)$ & $10(1.3)$ & $16(1.0)$ & \\
\hline $\mathrm{N}$ stage & & & & & $<0.001$ \\
\hline NO & $2192(76.8)$ & 408 (95.8) & 607 (77.4) & 1177 (71.6) & \\
\hline N1 & 351 (12.3) & $8(1.9)$ & 170 (21.7) & $173(10.5)$ & \\
\hline N2 & $310(10.9)$ & $10(2.3)$ & $7(0.9)$ & $293(17.8)$ & \\
\hline
\end{tabular}

Notes: $P^{\star}, \mathrm{P}$ value of Fisher exact test.

Abbreviations: USC, uterine serous cancer; LND, lymphadenectomy; PLND, pelvic lymphadenectomy; PALND, para-aortic lymphadenectomy; LNs, lymph nodes; PLNs, pelvic lymph nodes; SD, standard deviation. 


\begin{tabular}{|c|c|c|c|c|c|}
\hline \multirow[t]{2}{*}{ Characteristics } & Total & No LND & PLND only & $\begin{array}{l}\text { PLND + } \\
\text { PALND }\end{array}$ & $\begin{array}{l}P \\
\text { value }\end{array}$ \\
\hline & $\begin{array}{l}n=2853 \\
(\%)\end{array}$ & $\begin{array}{l}n=426 \\
(\%)\end{array}$ & $\begin{array}{l}n=784 \\
(\%)\end{array}$ & $n=1643(\%)$ & \\
\hline Grade & & & & & 0.04 \\
\hline G1 & $36(1.3)$ & $8(1.9)$ & $9(1.1)$ & $19(1.2)$ & \\
\hline G2 & $103(3.6)$ & $22(5.2)$ & $28(3.6)$ & $53(3.2)$ & \\
\hline G3 & $2146(75.2)$ & $293(68.8)$ & $588(75.0)$ & $1265(77.0)$ & \\
\hline Gx & $568(19.9)$ & $103(24.2)$ & $159(20.3)$ & $306(18.6)$ & \\
\hline Tumor size $(\mathrm{cm})$ & & & & & 0.003 \\
\hline$<2 \mathrm{~cm}$ & $524(18.4)$ & $91(21.4)$ & $153(19.5)$ & $280(17.0)$ & \\
\hline$\geq 2 \mathrm{~cm}$ & $1672(58.6)$ & $215(50.5)$ & $467(59.6)$ & $990(60.3)$ & \\
\hline Unknown & $657(23.0)$ & $120(28.2)$ & $164(20.9)$ & $373(22.7)$ & \\
\hline Regional LNs removed & & & & & $<0.001$ \\
\hline None & $426(14.9)$ & $426(100)$ & 0 & 0 & \\
\hline $1-16$ & $1189(41.7)$ & 0 & $612(78.1)$ & $577(35.1)$ & \\
\hline $16-100$ & $1238(43.4)$ & 0 & $172(21.9)$ & $1066(64.9)$ & \\
\hline PLNs removed & & & & & $<0.001$ \\
\hline None & $426(14.9)$ & $426(100)$ & 0 & 0 & \\
\hline $1-11$ & $1154(40.4)$ & 0 & $490(62.5)$ & $664(40.4)$ & \\
\hline $12-66$ & $1273(44.6)$ & 0 & $294(37.5)$ & $979(59.6)$ & \\
\hline Adjuvant therapy & & & & & $<0.001$ \\
\hline Combination & 1018 (35.7) & $86(20.2)$ & $256(32.7)$ & $676(41.4)$ & \\
\hline Chemotherapy alone & $871(30.5)$ & $148(34.7)$ & $241(30.7)$ & $482(29.3)$ & \\
\hline Radiation alone & $185(6.5)$ & $33(7.7)$ & $50(6.4)$ & $102(6.2)$ & \\
\hline None & 779 (27.3) & 159 (37.3) & $237(30.2)$ & $383(23.3)$ & \\
\hline
\end{tabular}

Notes: $P *, P$ value of Fisher exact test.

Abbreviations: USC, uterine serous cancer; LND, lymphadenectomy; PLND, pelvic lymphadenectomy; PALND, para-aortic lymphadenectomy; LNs, lymph nodes; PLNs, pelvic lymph nodes; SD, standard deviation. 


\begin{tabular}{|c|c|c|c|c|c|}
\hline \multirow[t]{2}{*}{ Characteristics } & Total & No LND & PLND only & $\begin{array}{l}\text { PLND + } \\
\text { PALND }\end{array}$ & \multirow[t]{2}{*}{$\begin{array}{l}P \\
\text { value }\end{array}$} \\
\hline & $\begin{array}{l}n=2853 \\
(\%)\end{array}$ & $\begin{array}{l}n=426 \\
(\%)\end{array}$ & $\begin{array}{l}n=784 \\
(\%)\end{array}$ & $n=1643(\%)$ & \\
\hline $\begin{array}{l}\text { Follow-up(mean } \pm S D \text {, } \\
\text { months) }\end{array}$ & $35.9 \pm 20.0$ & $\begin{array}{l}31.3 \pm \\
19.5\end{array}$ & $\begin{array}{l}35.7 \pm \\
20.1\end{array}$ & $37.2 \pm 20.0$ & $<0.001$ \\
\hline \multicolumn{6}{|c|}{ Notes: $P^{\star}, \mathrm{P}$ value of Fisher exact test. } \\
\hline \multicolumn{6}{|c|}{$\begin{array}{l}\text { Abbreviations: USC, uterine serous cancer; LND, lymphadenectomy; PLND, pelvic lymphadenectomy; } \\
\text { PALND, para-aortic lymphadenectomy; LNs, lymph nodes; PLNs, pelvic lymph nodes; SD, standard } \\
\text { deviation. }\end{array}$} \\
\hline
\end{tabular}

\section{Survival analysis based on the number of removed lymph nodes}

Patients who had $\geq 12$ pelvic lymph nodes removed had significantly better OS and CSS than those who had $<12$ pelvic lymph nodes removed or those without lymphadenectomy $(P<0.001$ for all, Fig. $1 A \& B)$. In the PLND + PALND group, there was no significant survival difference between patients who had $\geq 6$ para-aortic lymph nodes removed and those who had $<6$ removed (all $P>0.20$, Fig. 1C\&D).

The results of the univariate and multivariate analyses for the 1643 USC patients with PLND + PALND were similar to the findings of the above K-M survival analyses. As shown in Supplementary Table 2, the extent of PLND was a significantly independent prognostic factor for both OS and CSS ( $\geq 12$ subgroup vs< 12 subgroup, OS: HR 0.78, 95\% $\mathrm{Cl} 0.64-0.95, \mathrm{P}=0.02$; CSS: $\mathrm{HR} 0.75,95 \% \mathrm{Cl} 0.61-0.93, \mathrm{P}=0.008$ ), while the extent of PALND was not a significant prognostic factor.

\section{Independent prognostic factors for OS and CSS}

Age, grade, T stage, $\mathrm{N}$ stage, tumor size, lymphadenectomy, adjuvant therapy, regional lymph nodes removed and pelvic lymph nodes removed were significant prognostic factors in the univariate analysis as shown in Supplementary Table 3. All significant factors that met the proportional hazard assumption were entered into multivariate Cox regression analysis. The results revealed that age, $\mathrm{T}$ stage, $\mathrm{N}$ stage, tumor size, adjuvant therapy and pelvic lymph nodes removed were independent prognostic factors for both OS and CSS (all P<0.01, Table 2). 
Table 2

Multivariate COX regression analysis of OS and CSS.

\begin{tabular}{|c|c|c|c|c|}
\hline & os & & css & \\
\hline Characteristics & $\mathrm{HR}(95 \% \mathrm{Cl})$ & $P$ value & $\mathrm{HR}(95 \% \mathrm{Cl})$ & $P$ value \\
\hline \multicolumn{5}{|l|}{ Age } \\
\hline$<60$ & Reference & & Reference & \\
\hline$\geq 60$ & $1.42(1.14-1.77)$ & 0.002 & $1.39(1.09-1.75)$ & 0.007 \\
\hline \multicolumn{5}{|l|}{ T stage } \\
\hline T1 & Reference & & Reference & \\
\hline $\mathrm{T} 2$ & $1.76(1.42-2.19)$ & $<0.001$ & $1.96(1.55-2.49)$ & $<0.001$ \\
\hline T3 & $2.76(2.31-3.30)$ & $<0.001$ & $3.18(2.61-3.86)$ & $<0.001$ \\
\hline T4 & $3.77(2.65-5.38)$ & $<0.001$ & $4.59(3.17-6.64)$ & $<0.001$ \\
\hline \multicolumn{5}{|l|}{$\mathrm{N}$ stage } \\
\hline NO & Reference & & Reference & \\
\hline N1 & $1.86(1.52-2.27)$ & $<0.001$ & $1.85(1.49-2.29)$ & $<0.001$ \\
\hline N2 & $2.09(1.70-2.57)$ & $<0.001$ & $2.14(1.71-2.66)$ & $<0.001$ \\
\hline \multicolumn{5}{|l|}{ Tumor size (cm) } \\
\hline$<2$ & Reference & & Reference & \\
\hline$\geq 2$ & $1.76(1.38-2.24)$ & $<0.001$ & $1.69(1.29-2.21)$ & $<0.001$ \\
\hline Unknown & $1.24(0.94-1.62)$ & 0.12 & $1.27(1.71-2.66)$ & 0.11 \\
\hline \multicolumn{5}{|l|}{ Adjuvant therapy } \\
\hline Combination & Reference & & Reference & \\
\hline Chemotherapy alone & $1.25(1.05-1.49)$ & 0.01 & $1.25(1.03-1.51)$ & 0.02 \\
\hline Radiation alone & $1.79(1.33-2.41)$ & $<0.001$ & $1.72(1.24-2.39)$ & 0.001 \\
\hline None & $1.95(1.62-2.35)$ & $<0.001$ & $1.69(1.37-2.07)$ & $<0.001$ \\
\hline \multicolumn{5}{|l|}{ PLNs removed } \\
\hline None & Reference & $<0.001$ & Reference & \\
\hline $1-11$ & $0.57(0.47-0.69)$ & $<0.001$ & $0.62(0.50-0.77)$ & $<0.001$ \\
\hline
\end{tabular}




\begin{tabular}{|lllll|}
\hline \multicolumn{4}{|c|}{ OS } & CSS \\
\hline $12-66$ & $0.43(0.35-0.53)$ & $<0.001$ & $0.45(0.36-0.57)$ & $<0.001$ \\
\hline
\end{tabular}

Abbreviations: OS, overall survival; CSS, cancer-specific survival; $\mathrm{HR}$, hazard ratio; $\mathrm{Cl}$, confidence interval; PLNs, pelvic lymph nodes.

\section{Development and validation of a prognostic nomogram for OS and CSS}

A nomogram that incorporated the significant prognostic factors from the multivariate analysis was developed to predict 3- and 5-year OS and CSS, respectively (Fig. 2A\&B). The nomogram assigned a prognostic score for each variable on the point scale (Table 3). By summing the total score and locating it on the total point scale, we could easily predict 3 - and 5-year survival rates respectively. 
Table 3

Point assignment of prognostic factors and total scores.

\begin{tabular}{|c|c|c|c|c|}
\hline $\begin{array}{l}\text { Prognostic factors and total } \\
\text { scores }\end{array}$ & $\begin{array}{l}\text { Score } \\
(\mathrm{OS})\end{array}$ & $\begin{array}{l}\text { Predicted 5-year } \\
\text { OS (\%) }\end{array}$ & $\begin{array}{l}\text { Score } \\
\text { (CSS) }\end{array}$ & $\begin{array}{l}\text { Predicted 5-year } \\
\text { CSS (\%) }\end{array}$ \\
\hline \multicolumn{5}{|l|}{ Age(years) } \\
\hline$<60$ & 0 & & 0 & \\
\hline$\geq 60$ & 27 & & 21 & \\
\hline \multicolumn{5}{|l|}{ T stage } \\
\hline T1 & 0 & & 0 & \\
\hline $\mathrm{T} 2$ & 42 & & 44 & \\
\hline T3 & 76 & & 76 & \\
\hline $\mathrm{T} 4$ & 100 & & 100 & \\
\hline \multicolumn{5}{|l|}{$\mathrm{N}$ stage } \\
\hline NO & 0 & & 0 & \\
\hline N1 & 47 & & 40 & \\
\hline N2 & 55 & & 50 & \\
\hline \multicolumn{5}{|l|}{ Adjuvant therapy } \\
\hline Combination & 0 & & 0 & \\
\hline Chemotherapy alone & 17 & & 14 & \\
\hline Radiation alone & 44 & & 36 & \\
\hline None & 50 & & 34 & \\
\hline \multicolumn{5}{|l|}{ Tumor size (cm) } \\
\hline$<2$ & 0 & & 0 & \\
\hline$\geq 2$ & 42 & & 34 & \\
\hline Unknown & 16 & & 16 & \\
\hline \multicolumn{5}{|l|}{ PLNs removed } \\
\hline None & 64 & & 52 & \\
\hline $1-11$ & 21 & & 21 & \\
\hline
\end{tabular}

Abbreviations: OS, overall survival; CSS, cancer-specific survival; PLNs, pelvic lymph nodes. 


\begin{tabular}{|lllll|}
\hline $\begin{array}{l}\text { Prognostic factors and total } \\
\text { scores }\end{array}$ & $\begin{array}{l}\text { Score } \\
\text { (OS) }\end{array}$ & $\begin{array}{l}\text { Predicted 5-year } \\
\text { OS (\%) }\end{array}$ & $\begin{array}{l}\text { Score } \\
\text { (CSS) }\end{array}$ & $\begin{array}{l}\text { Predicted 5-year } \\
\text { CSS (\%) }\end{array}$ \\
\hline $12-66$ & 0 & & 0 \\
\hline Total scores (OS) & $>75$ & \\
\hline $0-84$ & $45-75$ & \\
\hline $85-161$ & $<45$ & & \\
\hline $162-$ max & & & \\
\hline Total scores (CSS) & & & $<5-80$ \\
\hline $0-69$ & & & $<55$ \\
\hline $70-140$ & & & \\
\hline 141 -max & & & \\
\hline Abbreviations: OS, overall survival; CSS, cancer-specific survival; PLNs, pelvic lymph nodes.
\end{tabular}

Internal validation was conducted for the nomogram with 2500 bootstrap resampling. The calibration curves demonstrated excellent consistency between the actual observation and nomogram prediction for 3- and 5-year survival probability (Fig. 3.A-D). The C-index for the primary cohort (OS: C-index 0.739, 95\% $\mathrm{Cl} 0.721-0.757$; CSS: C-index $0.752,95 \% \mathrm{Cl} 0.733-0.771$ ) was significantly higher than that of the FIGO staging system (OS: C-index $0.671,95 \% \mathrm{Cl} 0.653-0.689$; CSS: C-index $0.695,95 \% \mathrm{Cl} 0.676-0.713$; both $\mathrm{P}$ $<0.001$ ), and the average bootstrap-corrected C-index was 0.735 for OS and 0.752 for CSS. These results indicated this validated nomogram as a reliable prediction tool for both OS and CSS.

The patients were further divided into low-risk (predicted 5-year overall survival [Pos] $>75 \%$; predicted 5year cancer-specific survival [Pcss] $>80 \%$ ), intermediate-risk (45\% $\leq$ Pos $\leq 75 \% ; 55 \% \leq$ Pcss $\leq 80 \%$ ) and high-risk (Pos $<45 \%$; Pcss $<55 \%$ ) subgroups based on the 25th and 75th percentile values of the total scores (Table 3). As shown in Supplementary Fig. 2, these stratified risk subgroups demonstrated significant survival differences within each FIGO stage.

\section{Discussion}

Comprehensive lymphadenectomy is required for accurate staging in USC patients, but its therapeutic benefits are highly controversial. A large-scale randomized controlled trial in the treatment of endometrial cancer (ASTEC) has shown that systematic PLND offers no therapeutic benefits compared with no lymphadenectomy (22). In contrast, several retrospective studies have demonstrated that comprehensive lymphadenectomy was associated with significant improvements in the survival of both node-negative and node-positive women with USC $(21,23,25)$. These studies, however, had the following limitations. First, USC patients were generally underrepresented, thus the conclusion derived might not apply in USC 
patients. Second, most studies mentioned only the number of removed regional lymph nodes, while few explored the extension of PLND or the number of para-aortic lymph nodes. Our study enrolled 2843 USC patients who had undergone surgery from the SEER database. Among them, 1643 patients underwent PLND + PALND and the number of removed para-aortic lymph nodes was specified and analyzed. In our study, a larger number of removed pelvic lymph nodes was associated with better OS and CSS

(Fig. 1A\&B, Supplementary Table 1). However, the removal of more para-aortic lymph nodes did not lead to better OS and CSS (all $P>0.1$, Fig. 1C\&D).

The association between comprehensive PLND and survival can be interpreted in several ways. First, patients with a more extensive PLND may avoid understaging and therefore are more likely to receive adjuvant therapy when truly indicated. Nearly all USC patients after surgery should have received adjuvant chemotherapy \pm radiation therapy to further prolong survival $(4,17)$. However, our results showed that there were no significant differences in either the positive pelvic lymph node rate or the percentage of adjuvant therapy between the $\geq 12$ subgroup and $<12$ subgroup (both $P>$ 0.05 ,Supplementary Table 1). Therefore, the survival benefit of comprehensive PLND might not be explained simply by understaging or adjuvant therapy. Second, it may be possible that comprehensive PLND is therapeutic by removing more clinically occult lymph nodes and theoretically reducing the likelihood of lymph node recurrence. A multicenter retrospective cohort analysis in Korea with 476 surgically staged FIGO IB-IIIC2 patients indicated that more lymph nodes removed were associated with more positive lymph nodes detected and concluded that removing as many pelvic lymph nodes as possible improved survival in high-risk patients (18).

In our study, more extensive PALND did not improve OS or CSS (both $\mathrm{P}>0.2$, Fig. 1C\&D). The most likely explanation was the significantly higher complication rate and longer surgical time associated with comprehensive PALND. A population-based analysis from the National Cancer Database indicated that the addition of PALND improved survival in USC patients, however, they did not specify the respective number of pelvic and para-aortic lymph nodes. We believe that the significance of lymph node number from lymphadenectomy outweighs the significance of lymphadenectomy type, and the addition of PALND to PLND does not contribute to survival benefits when the number of removed pelvic lymph nodes is controlled. Therefore, we recommend PALND sampling over extensive PALND dissection, which would likely minimize perioperative morbidity while preserving staging accuracy.

Multiple prognostic factors can affect the survival of USC patients, but to our best knowledge, previous studies have never comprehensively integrated all relevant risk factors, and in particular, the number of removed para-aortic lymph nodes was rarely mentioned. Currently, the FIGO staging system remains the most widely used prognostic tool. However, as patient survival within the same FIGO stage is still heterogenous, we aim to develop a more comprehensive prognostic tool. Nomograms are intuitive statistical tools that can provide comprehensive and individualized survival predictions for cancer patients (30). Thus, in the current study, a nomogram was developed and validated with 2853 USC patients after surgery from the SEER database to predict the 3- and 5-year OS and CSS. 
Through univariate log-rank analysis and subsequent multivariate analysis, we identified age, $\mathrm{T}$ stage, $\mathrm{N}$ stage, tumor size, adjuvant therapy and the number of removed pelvic lymph nodes as independent prognostic factors for the survival of USC patients after surgery. Previously, younger age and adjuvant therapy were shown to be associated with better survival in high-risk endometrial cancer patients (8). In our study, patients older than 60 years had a significantly worse OS and CSS than those younger than 60 years old $(P<0.01$, Table 2$)$. Chemotherapy combined with radiotherapy led to better survival outcomes than chemotherapy alone, radiotherapy alone or neither. The FIGO staging system, which emphasizes on tumor invasion depth, lymph node involvement and the extent of tumor spread, has always been the standard survival prediction tool for gynecological cancer patients $(16,17)$. Similarly, we also identified $\mathrm{T}$ stage and $\mathrm{N}$ stage as the most important prognostic factors in the final nomogram. In addition, tumor size was found to be one of the key prognostic factors in our study. Generally, tumor size has been regarded as a factor indicating invasive ability without prognostic significance, but tumor size was not included in the current FIGO staging. However, a large National Cancer Database study with 2902 stage IIIC USC patients demonstrated that tumor size was associated with significantly worse survival ( $P$ $<0.0005)(19)$, which was consistent with our findings. We believe that USC patients with smaller tumor sizes are more likely to receive cytoreductive surgery with a higher success rate, which indirectly improves survival. In our study, calibration plots showed optimal consistency between the nomogram prediction and actual observation, which guaranteed the repeatability and reliability of the established nomogram. Excellent discrimination was demonstrated by the significantly higher $\mathrm{C}$-index of the nomogram than that of the FIGO staging system $(P<0.001)$. To further illustrate the independent discrimination ability of the nomogram, patients were divided into low-risk, intermediate-risk and high-risk subgroups based on the 25th and 75th percentile of the total scores (Table 3). In each FIGO stage, respective KM survival curves of different risk subgroups were plotted, as shown in Supplementary Fig. 2.

However, several limitations should be considered. First, our study is limited by the retrospective nature of the data and selective bias inevitably exists. Second, several recognized prognostic parameters were not available in the SEER database, including the tumor biomarkers CA125. Third, further external validation is warranted to confirm the prognostic utility and expand the application of our nomogram.

\section{Conclusion}

In conclusion, our study indicated that comprehensive PLND was significantly associated with better OS and CSS. However, comprehensive PALND did not convey survival benefits to USC patients. Therefore, we recommend comprehensive PLND combined with PALND sampling, which would likely minimize perioperative morbidity while preserving staging accuracy. In addition, we developed and validated a comprehensive and intuitive nomogram to better predict the 3- and 5-year OS and CSS of USC patients after surgery. Additional large-scale randomized controlled trials are warranted to validate our results.

\section{Declarations}

Availability of data and materials 
The datasets analyzed during this present study are available in publicly available SEER database. The data are also available from the corresponding author on reasonable request.

Acknowledgements

We sincerely thank the staff of the SEER program for their diligent effort and important work.

Funding

This work was funded by the National Science Foundation of China under Grant number 81702549 .

Ethics declarations

Ethics approval and consent to participate

Not applicable.

Consent for publication

Not applicable.

Competing interests

The authors declare that they have no competing interests.

Author contributions

Hui Li: Writing-original draft; Xiaofei Xie: Investigation; Yunyun Liu: Methodology; Xiaoxin Huang: Software; Haoliang Lin: Sources; Jing Li: Writing-review\&editing; Zhongqiu Lin: Conceptualization. All authors read and approved the final manuscript.

\section{Abbreviations}

USC, uterine serous cancer; SEER, Surveillance, Epidemiology and End Results; OS, overall survival; CSS, cause-specific survival; K-M, Kaplan-Meier; PLND, pelvic lymphadenectomy; PALND, para-aortic lymphadenectomy; FIGO, International Federation of Gynecology and Obstetrics; HRs, Hazard ratios; Cls, confidence intervals; IQR, interquartile range.

\section{References}

1. Moore KN, Fader AN. Uterine papillary serous carcinoma. CLIN OBSTET GYNECOL. [Journal Article; Review]. 2011 2011-06-01;54(2):278-91.

2. Wang Y, Yu M, Yang J, Cao D, Shen K, Lang J. Clinicopathological and survival analysis of uterine papillary serous carcinoma: a single institutional review of 106 cases. 2018;Volume 10:4915-28. 
3. Patsavas K, Woessner J, Gielda B, Rotmensch J, Yordan E, Bitterman P, et al. Optimal surgical debulking in uterine papillary serous carcinoma affects survival. GYNECOL ONCOL. 2011;121(3):5815 .

4. Del Carmen MG, Birrer M, Schorge JO. Uterine papillary serous cancer: A review of the literature. GYNECOL ONCOL. 2012;127(3):651-61.

5. Fader AN, Boruta D, Olawaiye AB, Gehrig PA. Uterine papillary serous carcinoma: epidemiology, pathogenesis and management. Current Opinion in Obstetrics and Gynecology. 2010;22(1):21-9.

6. Boruta DM, Gehrig PA, Fader AN, Olawaiye AB. Management of women with uterine papillary serous cancer: A Society of Gynecologic Oncology (SGO) review. GYNECOL ONCOL. 2009;115(1):142-53.

7. Hamilton CA, Cheung MK, Osann K, Chen L, Teng NN, Longacre TA, et al. Uterine papillary serous and clear cell carcinomas predict for poorer survival compared to grade 3 endometrioid corpus cancers. BRIT J CANCER. 2006 2006-01-01;94(5):642-6.

8. Acharya S, Hensley ML, Montag AC, Fleming GF. Rare uterine cancers. LANCET ONCOL. [Journal Article; Review]. 2005 2005-12-01;6(12):961-71.

9. Cham S, Huang Y, Tergas Al, Hou JY, Burke WM, Deutsch I, et al. Utility of radiation therapy for earlystage uterine papillary serous carcinoma. GYNECOL ONCOL. 2017;145(2):269-76.

10. Mahdi H, Nutter B, Abdul-Karim F, Amarnath S, Rose PG. The impact of combined radiation and chemotherapy on outcome in uterine papillary serous carcinoma compared to chemotherapy alone. J GYNECOL ONCOL. 2016;27(2).

11. Viswanathan AN, Macklin EA, Berkowitz R, Matulonis U. The importance of chemotherapy and radiation in uterine papillary serous carcinoma. GYNECOL ONCOL. 2011;123(3):542-7.

12. Hogberg T, Signorelli M, de Oliveira CF, Fossati R, Lissoni AA, Sorbe B, et al. Sequential adjuvant chemotherapy and radiotherapy in endometrial cancer-Results from two randomised studies. EUR $J$ CANCER. 2010;46(13):2422-31.

13. Maggi R, Lissoni A, Spina F, Melpignano M, Zola P, Favalli G, et al. Adjuvant chemotherapy vs radiotherapy in high-risk endometrial carcinoma: results of a randomised trial. $\mathrm{Br} \mathrm{J}$ Cancer. [Comparative Study; Journal Article; Randomized Controlled Trial; Research Support, Non-U.S. Gov't]. 2006 2006-08-07;95(3):266-71.

14. Huang $\mathrm{C}$, Tang $\mathrm{Y}$, Chiang $\mathrm{Y}$, Wang K, Fu H, Ke Y, et al. Impact of management on the prognosis of pure uterine papillary serous cancer - A Taiwanese Gynecologic Oncology Group (TGOG) study. GYNECOL ONCOL. 2014;133(2):221-8.

15. Rauh-Hain JA, Growdon WB, Schorge JO, Goodman AK, Boruta DM, McCann C, et al. Prognostic determinants in patients with stage IIIC and IV uterine papillary serous carcinoma. GYNECOL ONCOL. 2010;119(2):299-304.

16. Amant F, Mirza MR, Koskas M, Creutzberg CL. Cancer of the corpus uteri. INT J GYNECOL OBSTET. 2018;143(S2):37-50.

17. Koh WJ, Abu-Rustum NR, Bean S, Bradley K, Campos SM, Cho KR, et al. Uterine Neoplasms, Version 1.2018, NCCN Clinical Practice Guidelines in Oncology. J Natl Compr Canc Netw. [Journal Article; 
Practice Guideline]. 2018 2018-02-01;16(2):170-99.

18. Kim TH, Kim HS, Kim T, Chang S, Kim D, Ryu S, et al. Survival impact based on the thoroughness of pelvic lymphadenectomy in intermediate- or high-risk groups of endometrioid-type endometrial cancer: A multi-center retrospective cohort analysis. GYNECOL ONCOL. 2016;141(3):440-6.

19. Lin JF, Muniz K, Sukumvanich P, Gehrig P, Beriwal S, Kelley JL, et al. Survival advantage associated with multimodal therapy in women with node-positive (stage-IIIC) uterine papillary serous carcinoma: a National Cancer Database study. BJOG. [Evaluation Studies; Journal Article]. 2016 2016-1001;123(11):1846-52.

20. Alagkiozidis I, Weedon J, Grossman A, Wang P, Mize B, Wilson K, et al. Extent of lymph node dissection and overall survival in patients with uterine carcinosarcoma, papillary serous and endometrioid adenocarcinoma: A retrospective cohort study. INT J SURG. 2015;24:9-13.

21. Mahdi H, Kumar S, Al-Wahab Z, Ali-Fehmi R, Munkarah AR. Prognostic impact of lymphadenectomy in uterine serous cancer. BJOG. [Journal Article]. 2013 2013-03-01;120(4):384-91.

22. Kitchener H, Swart AM, Qian Q, Amos C, Parmar MK. Efficacy of systematic pelvic lymphadenectomy in endometrial cancer (MRC ASTEC trial): a randomised study. LANCET. [Journal Article; Multicenter Study; Randomized Controlled Trial; Research Support, Non-U.S. Gov't]. 2009 2009-0110;373(9658):125-36.

23. Smith DC, Macdonald OK, Lee CM, Gaffney DK. Survival impact of lymph node dissection in endometrial adenocarcinoma: a surveillance, epidemiology, and end results analysis. International Journal of Gynecologic Cancer. 2008;18(2):255-61.

24. Panici PB, Basile S, Maneschi F, Lissoni AA, Signorelli M, Scambia G, et al. Systematic Pelvic Lymphadenectomy vs No Lymphadenectomy in Early-Stage Endometrial Carcinoma: Randomized Clinical Trial. JNCI Journal of the National Cancer Institute. 2008 2008-12-03;100(23):1707-16.

25. Lutman CV, Havrilesky LJ, Cragun JM, Secord AA, Calingaert B, Berchuck A, et al. Pelvic lymph node count is an important prognostic variable for FIGO stage I and II endometrial carcinoma with highrisk histology. GYNECOL ONCOL. 2006;102(1):92-7.

26. Cragun JM, Havrilesky LJ, Calingaert B, Synan I, Secord AA, Soper JT, et al. Retrospective Analysis of Selective Lymphadenectomy in Apparent Early-Stage Endometrial Cancer. J CLIN ONCOL. 2005 2005-01-01;23(16):3668-75.

27. Todo Y, Kato H, Kaneuchi M, Watari H, Takeda M, Sakuragi N. Survival effect of para-aortic lymphadenectomy in endometrial cancer (SEPAL study): a retrospective cohort analysis. The Lancet. 2010 2010-01-01;375(9721):1165-72.

28. Balachandran VP, Gonen M, Smith JJ, DeMatteo RP. Nomograms in oncology: more than meets the eye. LANCET ONCOL. [Journal Article; Research Support, N.I.H., Extramural; Review]. 2015 2015-0401;16(4):e173-80.

29. Liang W, Zhang L, Jiang G, Wang Q, Liu L, Liu D, et al. Development and validation of a nomogram for predicting survival in patients with resected non-small-cell lung cancer. J CLIN ONCOL. [Journal 
Article; Multicenter Study; Research Support, Non-U.S. Gov't; Validation Study]. 2015 2015-0310;33(8):861-9.

30. Iasonos A, Schrag D, Raj GV, Panageas KS. How To Build and Interpret a Nomogram for Cancer Prognosis. J CLIN ONCOL. 2008 2008-03-10;26(8):1364-70.

\section{Supplemental Figures}

Supplementary Figure 1. Selection flow diagram.

Abbreviations: USC, uterine serous cancer; LND, lymphadenectomy; PLND, pelvic lymphadenectomy; PALND, para-aortic lymphadenectomy.

Supplementary Figure 2. Risk group stratifications within each FIGO stage. A-E are the overall survival curves (A, all patients; B-E, stage I-IVA), and F-J are the cause-specific survival curves ( $F$, all patients; G-J, stage I-IVA).

\section{Figures}


A

Strata + PLND=None + PLND=1-11 + PLND=12-66

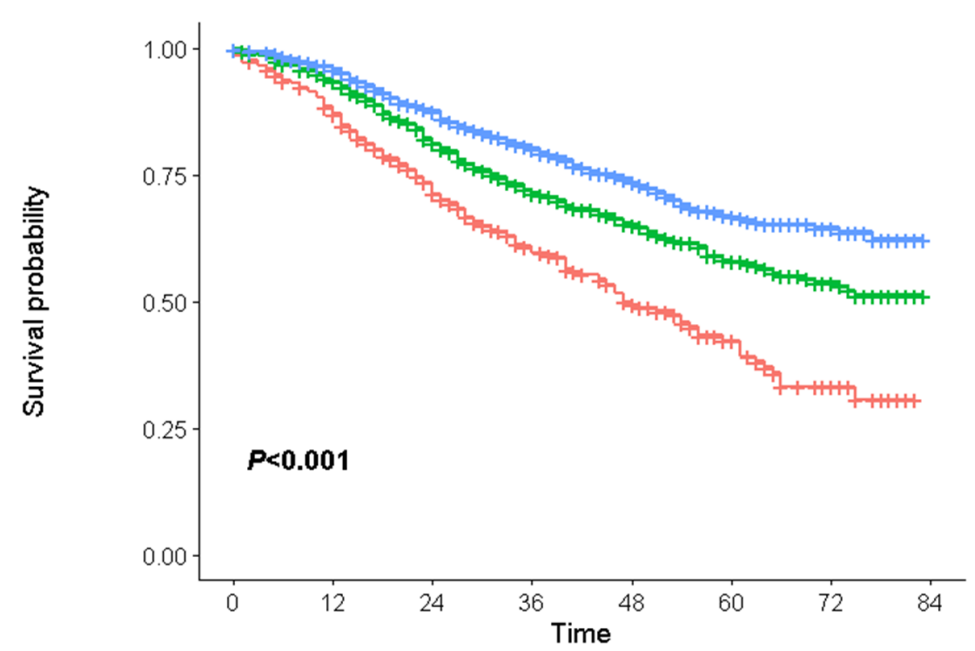

Number at risk

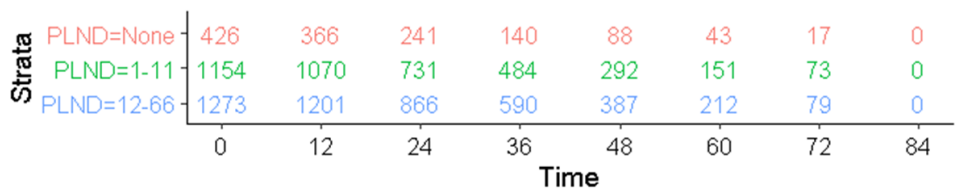

C
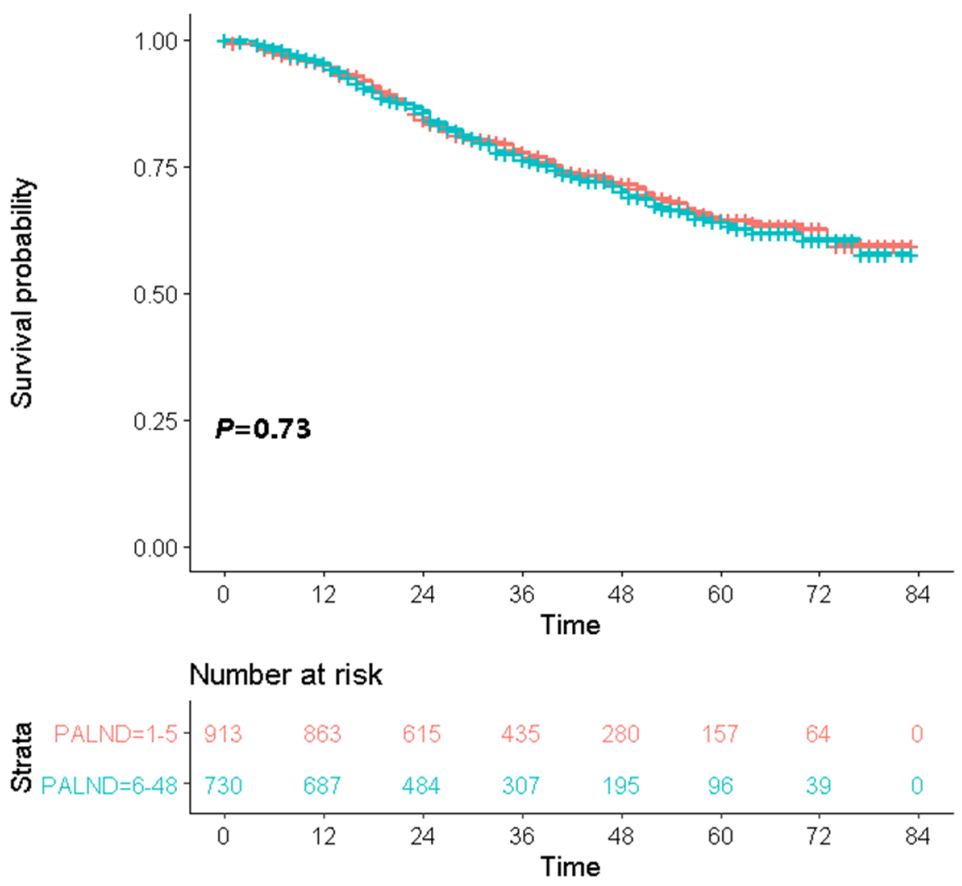

B

Strata + PLND=None + PLND=1-11 + PLND $=12-66$

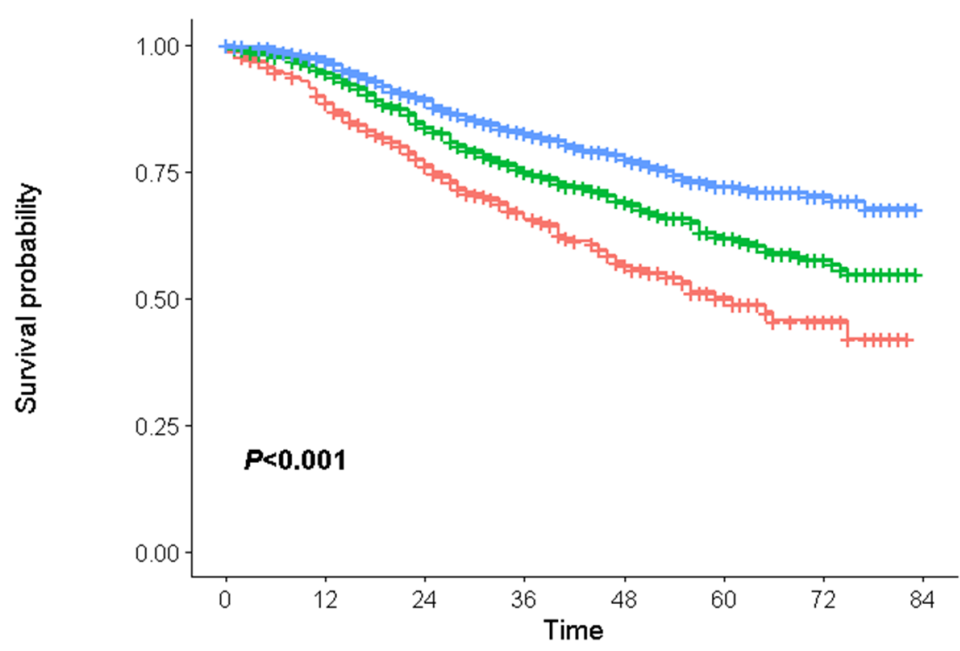

Number at risk

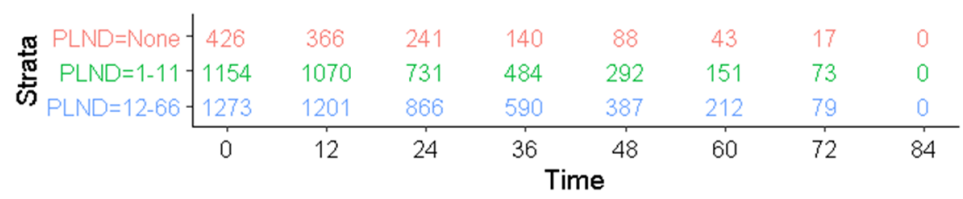

$\mathrm{D}$
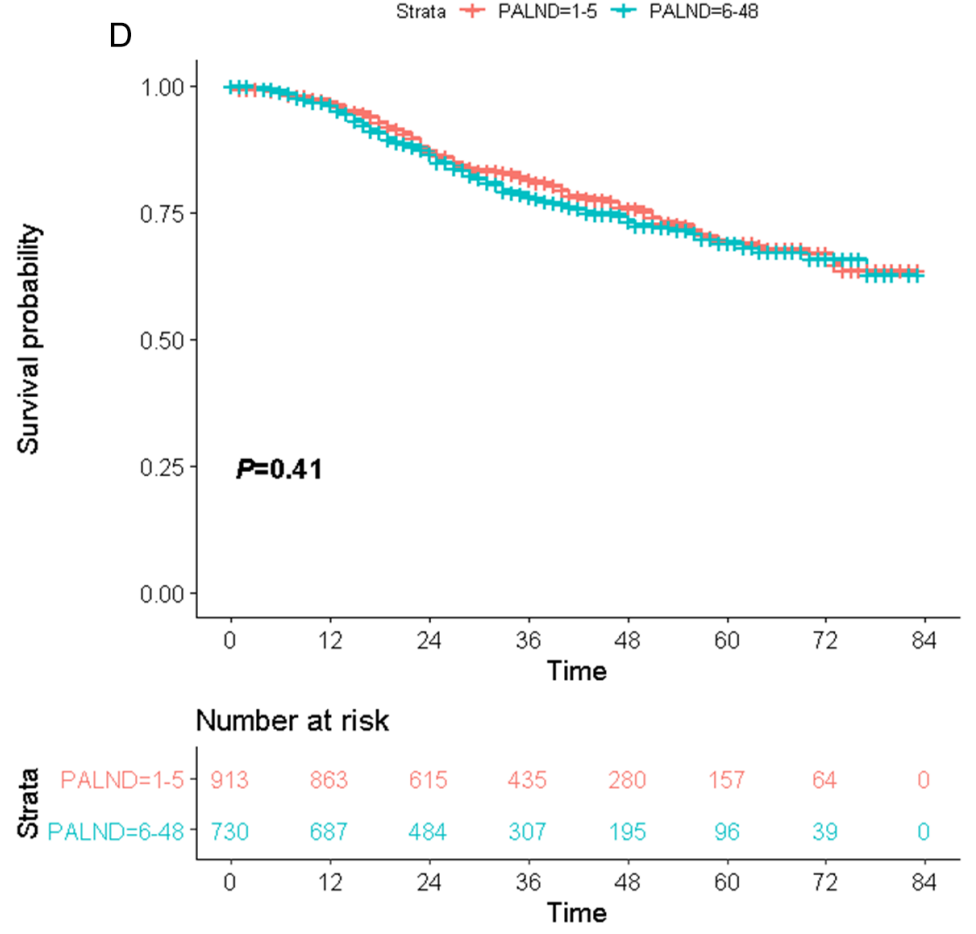

\section{Figure 1}

Kaplan-Meier survival curves for OS and CSS. (A) OS curve and (B) CSS curve for the 2853 USC patients with PLND who were divided into None, 1-11 and 12-66 subgroups. (C) OS curve and (D) CSS curve for the 1643 USC patients after PLND+PALND who were divided into 1-5 and 6-48 subgroups. Abbreviations: OS, overall survival; CSS, cause-specific survival; USC, uterine serous cancer; PLNs, pelvic lymph nodes; PLND, pelvic lymphadenectomy; PALNs, para-aortic lymph nodes; PALND, para-aortic lymphadenectomy. 

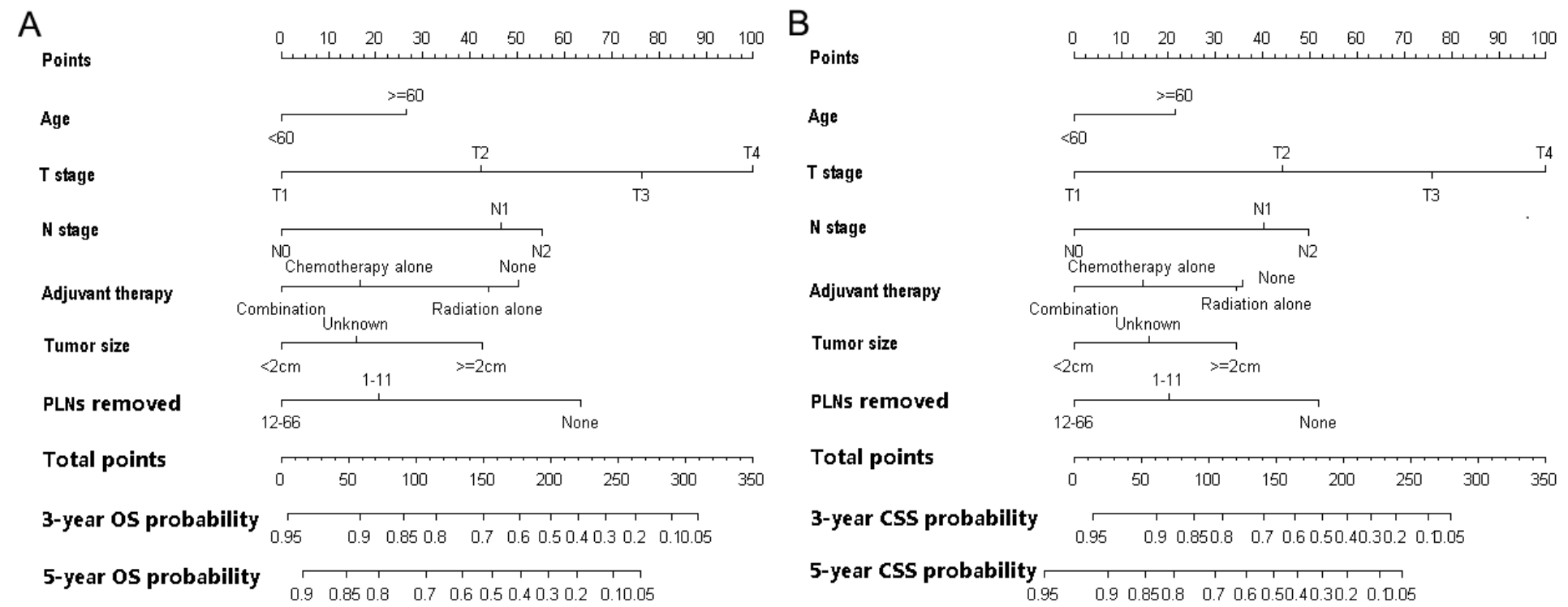

Figure 2

. Prognostic nomogram of OS and CSS for USC patients after surgery. Notes: A vertical line between each variable and point scale can be drawn to determine the points for each variable. The predicted survival rate was calculated according to the total points by drawing a vertical line from the total points scale to the 3- and 5-year survival scales. Abbreviations: OS, overall survival; CSS, cause-specific survival; USC, uterine serous cancer; PLNs, pelvic lymph nodes. 

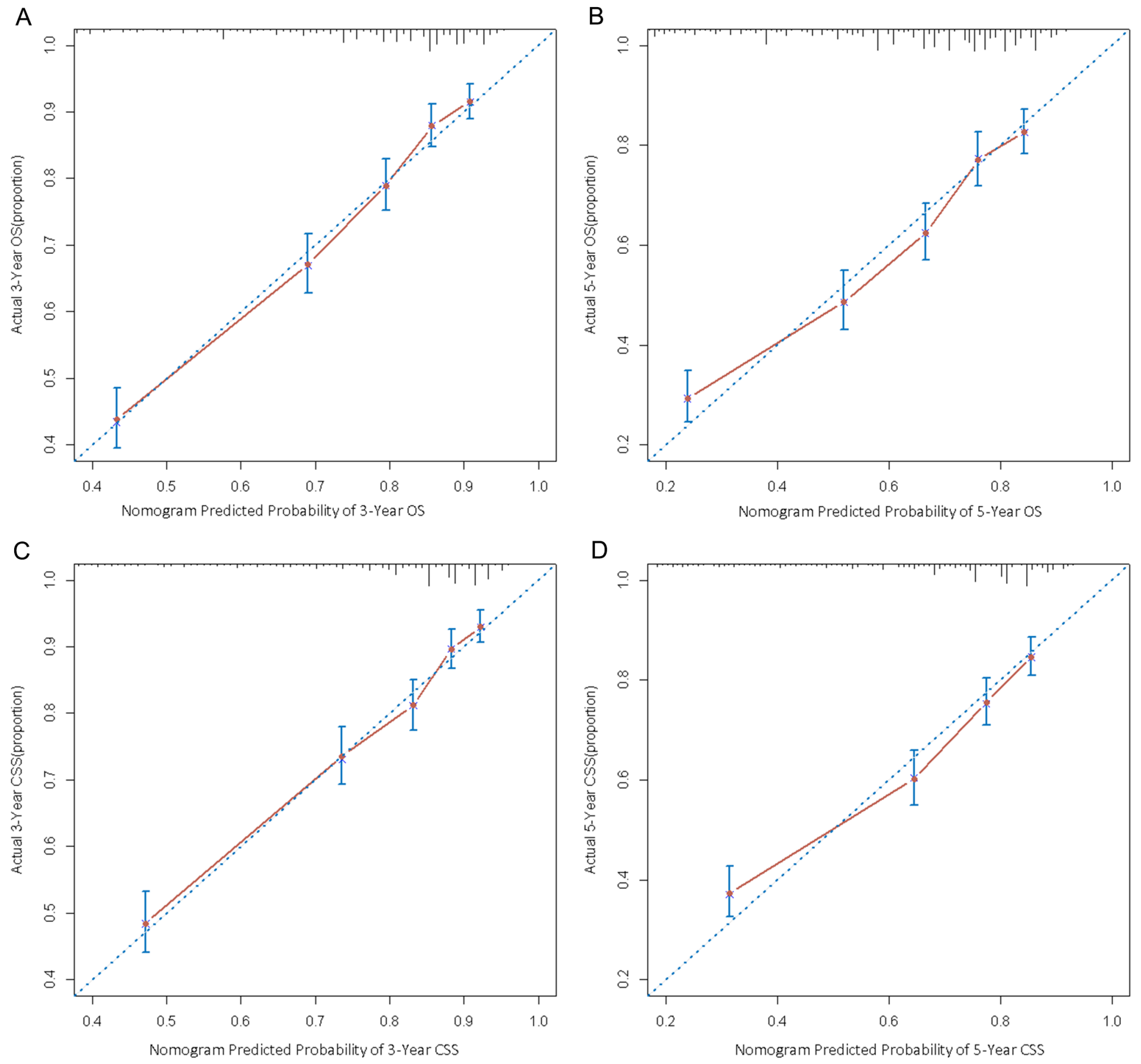

\section{Figure 3}

The internal calibration curves for predicting 3-year OS (A), 5-year OS (B), 3-year CSS (C) and 5-year CSS (D). Nomogram-predicted survival is plotted on the $x$-axis; actual survival is plotted on the $y$-axis. A plot along the 45-degree line indicates a perfect model in which the predicted probabilities are identical to the actual outcomes.

\section{Supplementary Files}

This is a list of supplementary files associated with this preprint. Click to download. 
- SupplementaryTable1.KeyvaluesbynumberofLNs.docx

- SupplementaryFigure2.Riskstratification.tif

- SupplementaryTable3.UnivariateCOXregression.docx

- SupplementaryFigure1.Selectiondiagram.tif

- SupplementaryTable2.Uniandmultiforsurgicalstaging.docx 